\title{
Quantitative Fluoroscopic Dose Saving in Cardiovascular Imaging with a Novel Motion Discriminating Temporal Filter
}

\author{
RM Manjeshwar ${ }^{1}$, PJ Dhawale ${ }^{2}$ \\ ${ }^{1}$ GE Global Research, Niskayuna, NY, USA \\ ${ }^{2}$ GE Healthcare, Buc, France
}

\begin{abstract}
Temporal recursive filters commonly used for fluoroscopic noise reduction blur moving objects and compromise device visibility. A motion discriminating temporal filter (MD-TF) has been developed for interventional applications on GE Innova Cardiovascular $X$-ray systems. This filter reduces motion-blur by detecting object motion and offers the potential for improved device visibility and/or dose savings. In this study, we quantified potential dose savings offered by the $M D$-TF using a realistic image synthesis model of the GE Innova digital flat panel system and visual perception experiments. The MD-TF applied to flat panel detector fluoroscopy images was found to offer up to $39 \%$ dose savings compared to unfiltered images. In comparison, a simple image lag resulted in no dose savings.

The results demonstrate the importance of optimal temporal filtering for maximizing device visibility, and for achieving maximum x-ray dose efficiency.
\end{abstract}

\section{Introduction}

Advances in device technology have led to a continuous improvement in percutaneous coronary interventions and associated outcomes. The growth in the number, complexity and duration of procedures has led to the need for greater performance in catheterization laboratories, especially when it comes to visualization of low contrast interventional objects like stents and guidewires. Due to the interventional nature of X-ray imaging, choices need to be made for a frame rate fast enough to meet visualization and latency requirements for hand-eye coordination, and dose per frame needed for low contrast object visibility. In large patients, the regulatory dose limit during fluoroscopy (typically $10 \mathrm{R} / \mathrm{min}$ ) often determines the maximum achievable image quality in raw images. Further enhancements are done with digital imaging processing. For adult cardiac procedures, frame rate ranging from $15-30 \mathrm{f} / \mathrm{s}$ is used clinically by the majority of cardiologists. Especially in coronary interventions, a sufficient frame rate is desired so that the motion of objects of interest is captured with high fidelity allowing tracking of objects during dynamic sequences.

Temporal image processing has proved its value in fluoroscopy for many years. Simple temporal recursive filtering has been extensively used in fluoroscopy of objects with minimal motion. It involves improving noise statistics in a static image by adding a fraction of the previous image to the currently acquired image. However, when simple recursive filters are applied on moving objects, they result in contrast degradation and a lag (moving objects with a trailing edge). Efficient temporal filtering in coronary imaging poses some interesting challenges. First, the objects of interest (guide-wires, stents, contrast filled arteries, etc.) are moving with the cardiac and respiratory cycles. Second, the anatomical background, which is also moving, is structured and has a wide dynamic range. Third, the clinicians prefer optimal visualization of interventional objects of interest, and have low tolerance for image lag.

The advent of high dynamic range digital flat panel detectors and advancements in real-time image processing hardware offer an excellent opportunity to introduce an intelligent scheme of achieving temporal averaging [1]. The acquisition technique (exposure parameters) control algorithm is capable of predicting image noise and minimal object contrast for commonly used interventional objects to a high degree of accuracy. In an intelligent scheme, the extent of temporal filtering can now be decided by a pixel-by-pixel difference signal along with an adaptive framework for controlling parameters of the recursive filter.

Our temporal filter is low-pass first-order recursive filter where each pixel is smoothed along the time domain as a function of estimated motion in that pixel. In the time domain, the output of the filter, $\mathrm{y}(\mathrm{n})$ is given by

$$
y(n)=y(n-1)+F\left(x(n)-y(n-1), A_{1}, A_{2}\right),
$$

where $\boldsymbol{x}(\boldsymbol{n})$ is the input value at the current time instant, and $\boldsymbol{A}_{1}$ and $\boldsymbol{A}_{2}$ are parameters that determine the filter strength and the sensitivity to motion detection. F indicates a mapping function. The output has a DC gain of one. The motion discriminator examines the difference 


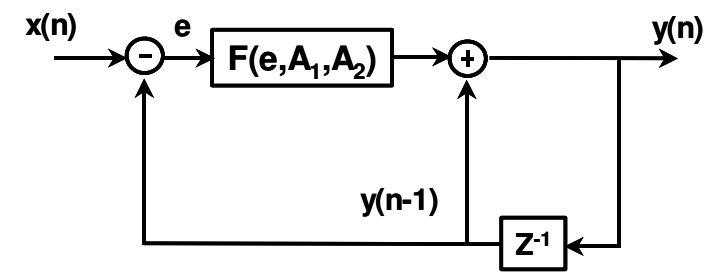

Figure 1: Temporal Filter Block Diagram

in the pixel intensities of successive image frames to estimate if motion has occurred in each pixel. The amount of filtering applied is accordingly modulated to smooth regions where motion has not occurred and preserve detail in regions of motion. Figure 2 illustrates the frequency response of the motion discrimination temporal filter where the pass band of the filter widens in regions of motion. In this study, we used a quantitative four alternative forced-choice (4-AFC) framework to assess the impact of control parameters on guide-wire object visibility and its implications for dose savings.

\section{Methods}

Many methods for image noise reduction filtering have been developed over the years but relatively little has been done in terms of quantitative evaluation of image quality as perceived by human observers. Typical metrics for evaluation of digital filters have been measures of noise variance reduction and image resolution. These metrics do not take into account the spatio-temporal frequency response of the human visual system and therefore poorly correlate with perceived image quality. An assessment of image quality should depend on the task to be performed based on those images. Since medical diagnosis and intervention often depends on the detection of low contrast objects, the ability of humans to detect low contrast targets in noisy images is a relevant metric of image quality.

The objective of our work was to quantify the x-ray dose savings afforded by the motion discriminating temporal filter relative to unfiltered fluoroscopic sequences for the task of guide-wire detection. We employed a 4-AFC detection paradigm [2] to measure the necessary $\mathrm{x}$-ray dose for observers to correctly detect the presence of a moving guide-wire 80 percent of the time for the unfiltered and filtered image sequences.

At a high level, in a 4-AFC-detection experiment, the observer is presented with four dynamic image sequences at a given frame rate side-by-side (see Figure 3), only one of which contains the guide-wire to be detected. The observer's objective is to select the panel which contains the guide-wire.

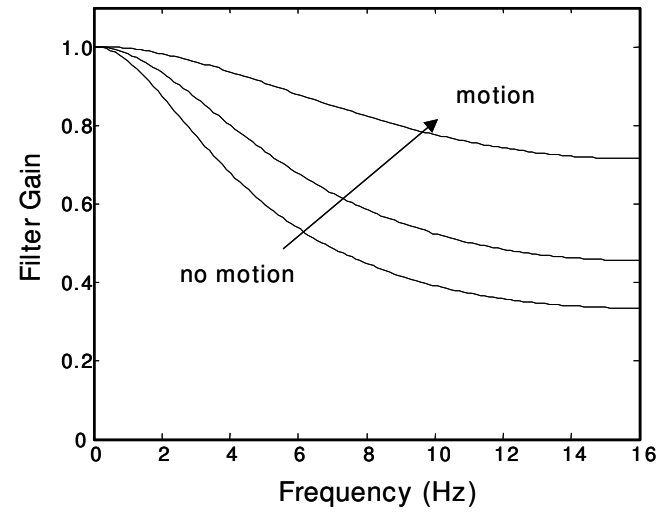

Figure 2: Temporal Frequency response

The x-ray exposure for the displayed image sequences (and resulting quantum noise) are adjusted using a maximum likelihood algorithm, over the course of many trials such that, at the experiment's conclusion, the x-ray exposure for which the observer achieves 80 percent detection accuracy is reliably identified.

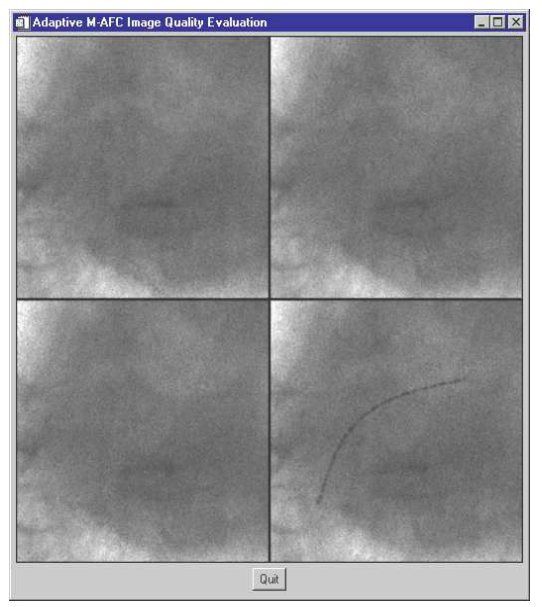

Figure 3: Observer has to identify the panel that contains the guide-wire. (Noise has been reduced in this image)

We followed the reference/test technique described in [4] to conduct our experiments. The quantum noise in the fluoroscopy sub-sequences was adapted so as to maintain observer performance at $80 \%$ probability of making the correct choice. For the reference experiment, four unfiltered image sequences (one with the guide-wire and three without the guide-wire) were randomly selected from a database of image sequences. The resultant sequences were then simultaneously displayed on a medical quality gray-scale monitor with the location of the guide-wire sequence randomly varied. The observer using a pointing device selected the sequence most likely to contain the guide-wire. Each reference trial was followed by a test trial, consisting of filtered image 
sequences. Interlacing the reference (unfiltered) and test (filtered) trials minimized observer effects such as fatigue, lapses in attention, or possible physiological changes that could occur during the course of the experiments [4]. Results were obtained after 100 reference trials interlaced with 100 test trials. Image noise (x-ray exposure) was determined from maximumlikelihood estimates, and standard errors were estimated by a method that accounted for adaptation.

\section{Image Generation}

The database of fluoroscopic image sequences required by the AFC experiments was generated using simulation tools that accurately modeled the physics and system properties of the GE Innova Flat Panel Cardiac imaging system.

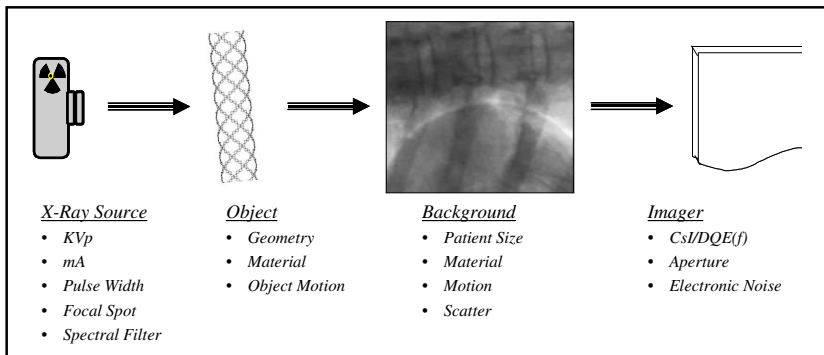

Figure 4: Synthetic image generation model

Image acquisition at 30 acquisitions/sec was simulated to synthesize clinically realistic image sequences that contain a 0.014 " guide-wire with right coronary motion on a dynamic anatomical background. To simulate the patient background, a selection of background attenuation patterns derived from high-dose ( $>30 \mu \mathrm{R} /$ acquisition) clinical cardiac image sequences were digitally filtered and converted to an equivalent acrylic thickness. Guidewires were generated digitally and were assigned material properties of steel to represent the shaft of the guide-wire. Coronary artery motion profiles were sampled from recorded clinical image sequences as a function of time and were applied to the guide-wire to simulate coronary motion. To produce a synthetic image sequence, $x$-ray spectrum at representative $\mathrm{KVp}$ was passed through the background and the guide-wire with realistic motion to modify the attenuation path. Detector and system properties, such as focal spot size, MTF, noise and scatter are applied to complete the simulation. Figure 4 shows the steps involved in this process. The model generates a sequence of dynamic images that reflects the impact of system parameters on the acquired images.

\section{Experiment Filter Parameters}

We compared human observer performance for the detection of a guide-wire in a fluoroscopic image sequence without filtering to image sequences with different parameters of the new FNR algorithm. We used the four different FNR filter parameter sets listed in Table 1 below. As explained in Section 2, the filter parameters A1 and A2 control the strength of the temporal filter and the motion discrimination window.

\begin{tabular}{|l|c|c|l|l|}
\hline & A1 & A2 & Filter Characteristic \\
\hline UNF & - & - & Unfiltered images \\
\hline FNR $_{1}$ & 0.20 & - & $20 \%$ Image lag, No motion sensitivity \\
\hline FNR $_{2}$ & 0.76 & 8 & Strong filtering, Strong motion sensitivity \\
\hline $\mathrm{FNR}_{3}$ & 0.50 & 8 & $\begin{array}{l}\text { Moderate filtering, Strong motion } \\
\text { sensitivity }\end{array}$ \\
\hline $\mathrm{FNR}_{4}$ & 0.50 & 15 & $\begin{array}{l}\text { Moderate filtering, Moderate motion } \\
\text { sensitivity }\end{array}$ \\
\hline
\end{tabular}

Table 1: Experimental Settings

\section{Results}

The mean exposure requirements for $80 \%$ probability of correct detection of the guide-wire before and after FNR filtering with four different parameter sets are shown in Figure 5. An analysis of variance (ANOVA) shows a significant effect $(\mathrm{p}<0.05)$ of filtering. Further, the Student-Neuman-Kuel (SNK) test shows a significant effect $(p<0.05)$ of filtering with FNR3. There was no significant difference $(\mathrm{p}>0.05)$ among the rest.

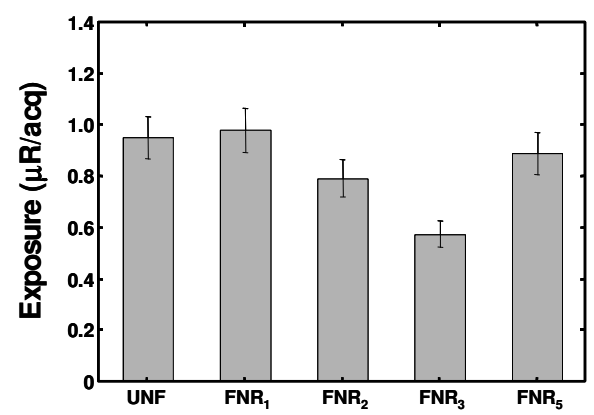

Figure 5: Exposure requirements for $80 \%$ probability of correct detection

The mean (across subjects) dose savings offered by the different FNR parameters when compared to no filtering are show in Figure 6. The maximum dose savings of $39 \%$ was achieved with medium strength and strong motion detection (FNR3). Dose savings of $16 \%$ and $8 \%$ were obtained with FNR2 and FNR4, respectively. 


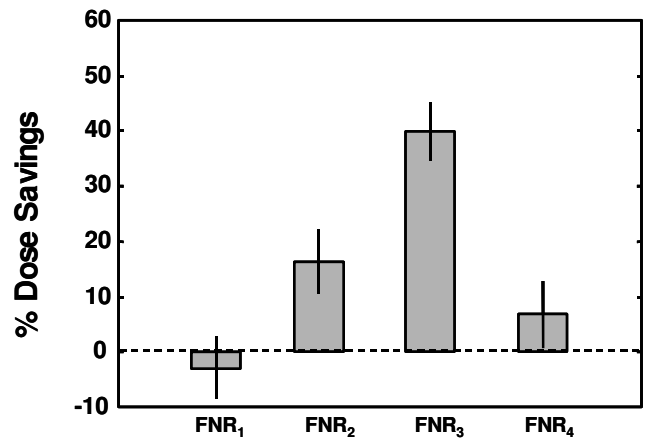

Figure 6: Percent X-ray Dose savings

\section{Discussion and conclusions}

There is a long history of quantitative evaluation of image quality in medical imaging [3]. In medical imaging, since diagnosis often depends on the detection of a low contrast object such as a lung nodule in a chest film, the ability of humans to detect low-contrast targets is often taken as a means of measuring image quality. At least three methods are commonly used. (1) receiver operating characteristic (ROC) experiments; (2) detection of a minimum contrast such as in a contrast detail phantom; (3) forced-choice experiments. We chose the four-alternative forced-choice method because it removes the subjectivity of an observer threshold, associated with the first two methods, and measures true target detectability on a statistical basis. If one translates that to $\mathrm{x}$-ray fluoroscopy, the task for the observer is to visualize and manipulate a guide-wire and not just detect it, an $80 \%$ probability of correct detection from a 4-AFC experiment might appear to be an inappropriate metric. However, it has been demonstrated that AFC experiments give similar results to the first two methods with the added advantage of experimental efficiency and reduced inter and intra observer variability [4].

The parameters for FNR1 simulate conventional temporal filtering with an image lag of $20 \%$. Conventional $\mathrm{x}$-ray angiography systems use $\mathrm{x}$-ray image intensifiers coupled to analog cameras. Unlike the new solid-state detector simulated here, which has a shortterm fluoroscopic lag of the order of $1-2 \%$, x-ray image intensifiers coupled to analog cameras have as much as $20 \%$ image lag. The present experimental data argues that the amount of low-pass temporal filtering obtained either with conventional temporal filters (lag of 20\%) or with analog camera based system negligibly affects detectability. Wilson et al. modeled human observer detection of stationary targets in temporally filtered sequences [4]. They report that, in order to substantially impact detectability with temporal filtering, one must attenuate noise at temporal frequencies below the range where the visual-system filter dominates. Digital temporal filters should be designed with this important factor in mind.

Maximum dose savings were obtained with moderate filtering and strong motion sensitivity. However, strong motion sensitivity coupled with strong filtering degraded detection. Detection was also degraded with moderate motion sensitivity. We hypothesize that despite the strong motion detection, the strong temporal filtering resulted in some motion blur as the noise-free contrast of the steel guide-wire prior to filtering was marginally above the threshold of motion detection.

The results are encouraging and provide a framework for assessing the impact of image processing parameters on threshold object visibility, and hence the required radiation dose. With the best set of parameters for the optimized FNR algorithm on GE Innova systems, we were able to achieve dose savings as much as $39 \%$ for equivalent object visibility. This is a substantial improvement, and indicates the magnitude of impact a well designed motion discriminating temporal filter can have on dose and object visibility. In large patients, the clinician is often operating in a regulatory dose limited environment. Once the skin dose reaches a regulatory limit, the detector entrance exposure (and hence the image quality) begins to drop exponentially with increasing patient thickness. Selection of optimal image processing parameters with the motion discriminating temporal filter will yield superior results in such difficult imaging situations.

\section{References}

[1] Dhawale PJ, Lebihen T, and Kautz GM, "Method and apparatus for performing fluoroscopic noise reduction." US Patent Number 6,314,160 B1, 2001

[2] Xue P, Thomas C. W, Gilmore G. C, and Wilson D. L, "An adaptive reference/test paradigm: Application to pulsed fluoroscopy perception," Behav. Res. Meth., Instrum. Comput., 1998, 30:332-348.

[3] Burger G. C. E, "The perceptibility of details in Roentgen examinations of the lung," Acta Radiol., 1949, 31:193-222.

[4] Wilson D.L, Jabri K.N and, Manjeshwar R.M, "Quantitative Image Quality Studies and the Design of Xray Fluoroscopy Systems" Physics and Psychophysics Vol. I., SPIE Press 2000

Address for correspondence

Paritosh Dhawale, PhD

283 Rue de la Miniere; BP 34 ; 78533 Buc Cedex, France 